\title{
Study of the Clinical Characteristics and Management of Indian Outpatients of Chronic Heart Failure with Coexistent Diabetes Versus those without Diabetes (HF-DIAB): Rationale and Design
}

\section{Meena Lopez ${ }^{1,4 *}$, Jamshed Dalal ${ }^{2}$, Sundeep Salvi ${ }^{3}$ and Jaideep Gogtay ${ }^{4}$}

${ }^{1}$ Department of Health and Biological Sciences, Symbiosis International University (SIU), Pune, Maharashtra, India

${ }^{2}$ Department of Cardiac Sciences, Kokilaben Dhirubhai Ambani Hospital, Mumbai, Maharashtra, India

${ }^{3}$ Chest Research Foundation, Pune, Maharashtra, India

${ }^{4}$ Department of Medical Affairs, Cipla Ltd., Mumbai, Maharashtra, India

\begin{abstract}
Introduction: Heart failure (HF) is a significant and rising health burden in India. Diabetes mellitus is commonly prevalent in HF patients. However, data comparing the clinical characteristics and management of Indian $\mathrm{HF}$ patients with and without coexistent diabetes is lacking. Also, differences in HF management patterns between cardiologists and internists have not been systematically ascertained in India. This study is designed to address these gaps in knowledge.
\end{abstract}

Methods and analysis: HF-DIAB is a prospective multicenter study that aims to obtain data on 2,500 Indian adult outpatients with chronic HF recruited from 250 outpatient clinics across India; half of the included subjects will have a history of coexistent diabetes. Data on the patient's demographics, personal and medical history, signs and symptoms of $\mathrm{HF}$, etiology and clinical presentation of HF, diagnostic tests performed, previous HF-related hospitalizations, drugs prescribed for $\mathrm{HF} /$ diabetes and other comorbidities will be collected. Independent sample t-test will be used to compare differences between diabetic and non-diabetic HF subjects for various clinical characteristics measured using clinical variables. Chi- square test will be used to find out the association between diabetics and non-diabetics with various risk factors and clinical characteristics measured using categorical data. Differences in HF treatment, as per the specialty of the treating doctor, will also be compared using Chi-square test.

Ethics and dissemination: The study documents have been reviewed and approved by an independent ethics committee. Data-sharing consent will be obtained from each patient before enrollment in the study. The study results will be disseminated through publications in peer-reviewed journals.

Trial registration number: Clinical Trials Registry: India [CTRI/2017/07/009069].

Keywords: Heart failure; Diabetes; HF-DIAB; India

Abbreviations: HF: Heart Failure; EF: Ejection Fraction; CHD: Coronary Heart Disease; RHD: Rheumatic Heart Disease; ACE: Angiotensin Converting Enzyme; ARB: Angiotensin Receptor Blocker; ARNI: Angiotensin Receptor Neprilysin Inhibitor; MRA: Mineralocorticoid Receptor Antagonist; HFrEF: Heart Failure with Reduced Ejection Fraction; HFpEF: Heart Failure with Preserved Ejection Fraction; CV: Cardiovascular; BMI: Body Mass Index; SBP: Systolic Blood Pressure; LVEF: Left Ventricular Ejection Fraction; RAAS: Renin Angiotensin Aldosterone System; DCF: Data Capture Form; NYHA: New York Heart Association

\section{Introduction}

Heart failure (HF) is estimated to affect 26 million people worldwide [1]. It is characterized by functional or structural cardiac abnormalities resulting in a reduced cardiac output and/or elevated intracardiac pressures at rest or during stress [2]. It is commonly classified as HF with reduced ejection fraction $(\mathrm{EF} \leq 40 \%)$ and $\mathrm{HF}$ with preserved ejection fraction $(\mathrm{EF} \geq 50 \%)$ [2]. Owing to the absence of adequate surveillance systems, reliable prevalence estimates of HF are lacking for India. While one model based on estimates of coronary heart disease (CHD), hypertension, diabetes and rheumatic heart disease as the main causes of HF has conservatively estimated the number of people living with HF in India in the range of 1.3 to 4.6 million, another estimate pegs it at 8-10 million individuals [3,4]. Importantly, the burden of HF is bound to rise in India due to the aging of the population, increasing burden of hypertension, CHD and diabetes mellitus, and the persistence of diseases such as rheumatic heart disease (RHD) and non-ischemic cardiomyopathies $[3,5]$.

Despite the growing burden, data on various aspects related to $\mathrm{HF}$ in India are sparse. We plan to conduct the HF-DIAB (Study of the Clinical Characteristics and Management of Indian Outpatients of Chronic Heart Failure with Coexistent Diabetes Vs. those without Diabetes) to generate data on two key areas concerning HF patients: HF with coexistent diabetes and optimization of HF management.

\section{HF-DIAB Study Rationale}

\section{Suboptimal management of HF: A serious concern}

The standard of care for all patients with HF with reduced ejection

*Corresponding author: Meena Lopez, Department of Medical Affairs, Cipla Ltd., Mumbai, Maharashtra, India, Tel: 9820515620; E-mail: meena@cipla.com

Received March 15, 2018; Accepted April 10, 2018; Published April 13, 2018

Citation: Lopez M, Dalal J, Salvi S, Gogtay J (2018) Study of the Clinical Characteristics and Management of Indian Outpatients of Chronic Heart Failure with Coexistent Diabetes Versus those without Diabetes (HF-DIAB): Rationale and Design J Cardiovasc Dis Diagn 6: 318. doi: 10.4172/2329-9517.1000318

Copyright: ( 2018 Lopez M, et al.. This is an open-access article distributed under the terms of the Creative Commons Attribution License, which permits unrestricted use, distribution, and reproduction in any medium, provided the original author and source are credited. 
Citation: Lopez M, Dalal J, Salvi S, Gogtay J (2018) Study of the Clinical Characteristics and Management of Indian Outpatients of Chronic Heart Failure with Coexistent Diabetes Versus those without Diabetes (HF-DIAB): Rationale and Design. J Cardiovasc Dis Diagn 6: 318. doi: 10.4172/2329-9517.1000318

Page 2 of 6

fraction (HFrEF) should include an angiotensin converting enzyme (ACE) inhibitor [which can be substituted with an angiotensin receptor blocker (ARB) or an angiotensin receptor neprilysin inhibitor (ARNI) in appropriate patients] and a beta-blocker, unless contraindicated [6,7]. Mineralocorticoid receptor antagonists (MRAs: spironolactone/ eplerenone) are also indicated in patients who continue to remain symptomatic despite receiving the abovementioned classes of drugs $[6,7]$. Interventions such as percutaneous coronary intervention (PCI) and coronary artery bypass grafting (CABG), and devices [cardiac resynchronisation therapy (CRT), implantable cardioverter defibrillator (ICD)] are recommended in appropriately selected HF patients as per guidelines [6,7]. In HF patients with preserved ejection fraction (HFpEF), no treatment has been shown to conclusively reduce morbidity and mortality; hence the main aim in these patients is to reduce symptoms, manage risk factors and improve quality of life [6].

Adherence to guideline-directed therapies by physicians has been shown to be associated with improved clinical outcomes. In the prospective, longitudinal QUALIFY (QUality of Adherence to guideline recommendations for LIFe-saving treatment in heart failure surveY) study conducted in 36 countries in Asia, Australia, Europe, Africa, Middle East and America, and involving 6669 outpatients with HFrEF, poor adherence to guideline-directed therapy was shown to be associated with increased risk of total mortality, cardiovascular (CV) mortality, and HF mortality (Table 1) [8]. Similarly, findings from the SUGAR (SUrvey of Guideline Adherence for Treatment of Systolic Heart Failure in Real World) trial which followed up 1297 HFrEF patients showed that prescription of guideline-directed therapy at discharge had a significant impact on event-free survival rate of mortality and re-hospitalization $(94.7 \%$ vs. $89.8 \%, p=0.003$ and $62.3 \%$ vs. $56.4 \%, \mathrm{p}=0.041$, respectively for the good vs poor guideline-adherence groups) [9].

Unfortunately, available published data suggest that the vast majority of HF patients in India do not receive these guidelinedirected therapies. In the hospital-based Trivandrum Heart Failure Registry, which followed 1,205 HF patients enrolled from 18 hospitals in Trivandrum, only $25 \%$ patients received guideline-directed medical therapy at discharge [10]. Importantly, the study also showed that suboptimal adherence to guideline-directed medical therapy was associated with an increased risk of mortality and hospital readmissions. The underuse of evidence-based therapies has been observed among HF outpatients as well. Data from the American College of Cardiology's PINNACLE India Quality Improvement Program (PIQIP) registry which enrolled 15,870 HFrEF patients from 10 centers in India, showed that usage of both beta-blockers and ACE inhibitors/ARBs was documented in only $30 \%$ patients [11].

A Polish study, which prospectively compared differences in the management of HF outpatients between cardiologists $(\mathrm{N}=500)$ and generalists $(\mathrm{N}=290)$ showed that the former group was more likely to prescribe an ACE inhibitor/ARB, an MRA and a beta-blocker than

\begin{tabular}{|c|c|c|c|}
\hline Parameter & $\begin{array}{c}\text { Baseline } \\
\text { Adherence Score }\end{array}$ & $\begin{array}{l}\text { Hazard Ratio } \\
(95 \% \mathrm{Cl})\end{array}$ & P, Group Effect \\
\hline \multirow{2}{*}{ Total mortality } & Moderate vs. good & $1.85(1.26 ; 2.72)$ & \multirow{2}{*}{0.001} \\
\hline & Poor vs. good & $2.21(1.42 ; 3.44)$ & \\
\hline \multirow{2}{*}{ CV mortality } & Moderate vs. good & $2.06(1.32 ; 3.20)$ & \multirow{2}{*}{0.003} \\
\hline & Poor vs. good & $2.27(1.37 ; 3.77)$ & \\
\hline \multirow{2}{*}{ HF-mortality } & Moderate vs. good & $1.84(1.07 ; 3.18)$ & \multirow{2}{*}{0.032} \\
\hline & Poor vs. good & $2.26(1.21 ; 4.20)$ & \\
\hline
\end{tabular}

Table 1: Impact of adherence score on 6-month clinical outcomes, as per multivariate analysis. the latter (59\% vs 51\%) [12]. An Australian study based on patient admissions for HF showed that patients admitted by a cardiologist were more likely to be on beta-blockers and spironolactone at discharge [13]. The Euro Heart Failure Survey which screened the discharge data of HF patients admitted to 115 hospitals from 24 countries showed similar results: patients in cardiology wards were more likely to receive an ACE inhibitor (71.5\% vs $56.4 \%$ ) or a beta-blocker (50.7\% vs $26.3 \%$ ) as compared with those in a general internal medicine ward [14]. However, no study has systematically ascertained differences in the management of HF patients between cardiologists and internists (the two categories of doctors who primarily treat HF) in India.

\section{Diabetes: A significant comorbidity in HF}

Diabetes is an important and common comorbidity in HF patients, with the prevalence ranging from $10 \%$ to $47 \%$, depending on the cohort studied [15]. Approximately $15 \%-35 \%$ of HF patients have been reported to have concomitant diabetes in randomized clinical trials [16]. The PIQIP registry and the Trivandrum HF Registry reported diabetes to be coexistent in $23 \%$ and $55 \%$ of Indian HF patients, respectively $[10,11]$. Notably, the INTER-CHF study reported coexistent diabetes to be more prevalent among Indian HF patients as compared with HF patients in Africa and South America (26\% vs. $17 \%$ and $21 \%$, respectively) [17]. The ASIAN-HF (Asian Sudden Cardiac Death in Heart Failure) registry too reported a higher prevalence of coexistent diabetes among Indian ethnicity patients as compared with Chinese, Japanese, Korean, Thai and Filipino ethnicities $(42.1 \%$ as compared with $40.9 \%, 31.7 \%, 30.2 \%, 34.5 \%$ and $27.7 \%$, respectively) [18].

The presence of diabetes as a comorbidity in HF patients is clinically relevant since studies have shown that HF patients may differ in their clinical characteristics as compared with those without diabetes in terms of their age, etiology, EF and presence of comorbidities [19-22]. Analysis of patients with HFpEF included in the UK-based Digitalis Investigation Group (DIG) ancillary study showed that HF patients with coexistent diabetes were younger, more likely to be female, had a higher body mass index (BMI), systolic blood pressure (SBP), pulse pressure and heart rate, and were more likely to have peripheral edema and pulmonary congestion as compared with those without coexistent diabetes (Table 2) [19]. A prior history of hypertension and ischemic etiology of HF was also more commonly seen in these patients [19]. A UK population-based cohort study reported that as compared with HF patients without coexistent diabetes, those with coexistent diabetes had a greater prevalence of comorbidities such as hypertension, prior stroke, vascular disease and ischemic heart disease [20]. The OPTIMIZE-HF (Organized Program to Initiate Lifesaving Treatment in Hospitalized Patients with Heart Failure) registry which evaluated 48,612 hospitalized patients with HF showed similar results: HF patients with diabetes tended to be younger, had a greater likelihood of ischemic etiology, hyperlipidemia and a hypertension history, had a higher left ventricular ejection fraction (LVEF) and were more likely to have edema as compared with those without diabetes [21]. A study conducted in China showed that as compared with diabetic HF patients, non-diabetic HF patients were older, had a higher weight, increased risk of hospitalization, a higher SBP, a lower hemoglobin and a lower EF (Table 3) [22].

Notably, the presence of comorbid diabetes has been shown to worsen the prognosis of the HF patient $[21,23,24]$. In the multinational (21 European and Mediterranean countries) ESC-HFA (European Society of Cardiology and ESC-Heart Failure Association) Registry, which evaluated 9,428 outpatients with HF, those with coexistent diabetes had higher cumulative incidence rates of all-cause mortality 
Citation: Lopez M, Dalal J, Salvi S, Gogtay J (2018) Study of the Clinical Characteristics and Management of Indian Outpatients of Chronic Heart Failure with Coexistent Diabetes Versus those without Diabetes (HF-DIAB): Rationale and Design. J Cardiovasc Dis Diagn 6: 318. doi: 10.4172/2329-9517.1000318

Page 3 of 6

\begin{tabular}{|c|c|c|}
\hline Parameter & HF with Diabetes & HF without Diabetes \\
\hline Age (years) & $64.8 \pm 9.8^{* *}$ & $67.6 \pm 10.4$ \\
\hline Female & $48.8 \%^{\#}$ & $38.00 \%$ \\
\hline BMI (kg/m $)$ & $30.8 \pm 7.3^{* *}$ & $27.7 \pm 5.5$ \\
\hline $\begin{array}{c}\text { Heart rate (beats per } \\
\text { minute) }\end{array}$ & $77.7 \pm 12.4^{*}$ & $75.0 \pm 11.7$ \\
\hline \begin{tabular}{c} 
SBP $(\mathrm{mm} \mathrm{Hg})$ \\
\hline $\begin{array}{c}\text { Brachial pulse pressure } \\
\text { (mm Hg) }\end{array}$
\end{tabular} & $141.4 \pm 21.8^{* *}$ & $136.0 \pm 20.9$ \\
\hline $\begin{array}{c}\text { Peripheral edema } \\
\text { Radiologic evidence of } \\
\text { pulmonary congestion }\end{array}$ & $64.8 \pm 18.5^{* *}$ & $59.0 \pm 18.1$ \\
\hline Ischemic HF etiology & $68.5 \%^{* *}$ & $55.00 \%$ \\
\hline Hypertension & $70.9 \%^{* *}$ & $60.00 \%$ \\
\hline
\end{tabular}

${ }^{*} \mathrm{p}=0.01 ;{ }^{* *} \mathrm{p} \leq 0.001 ; \# \mathrm{p}=0.002$

Continuous variables are presented as means \pm standard deviations; categorical variables presented as percentages.

BMI: Body Mass Index; SBP: Systolic Blood Pressure; HF: Heart Failure.

Table 2: Comparison of clinical and demographic parameters between HF patients with and without diabetes: DIG ancillary study.

\begin{tabular}{|c|c|c|}
\hline Parameter & HF with Diabetes & HF without Diabetes \\
\hline Age (years) & $67.77 \pm 12.39^{\star}$ & $64.13 \pm 15.96$ \\
\hline Weight (kg) & $68.29 \pm 14.13^{* *}$ & $64.69 \pm 12.06$ \\
\hline Hospitalization (n) & $1.86 \pm 2.26^{* * *}$ & $1.48 \pm 1.28$ \\
\hline $\mathrm{SBP}(\mathrm{mmHg})$ & $133.33 \pm 20.83^{*}$ & $128.45 \pm 23.42$ \\
\hline Hemoglobin (g/L) & $125.12 \pm 21.61^{*}$ & $130.75 \pm 21.40$ \\
\hline LVEF (\%) & $37 \pm 8^{\#}$ & $38 \pm 8$ \\
\hline
\end{tabular}

Table 3: Comparison between HF patients with diabetes and without diabetes: study from China.

(9.4\% vs $7.2 \%$; hazard ratio (HR) 1.28$)$, CV mortality ((4.8\% vs $3.8 \%$; HR 1.28) and first hospitalization for worsening HF (13.8\% vs $9.3 \%$; HR 1.37) at 1 year [24]. Another study which prospectively followed 1091 outpatients with HFrEF showed similar results: there was an approximate doubling of the risk of total and CV mortality in $\mathrm{HF}$ patients with diabetes as compared with those without diabetes, irrespective of the etiology (ischemic/non-ischemic) of HF [23].

The worse prognosis highlights the need for aggressive management in this subgroup of HF patients. However, a retrospective audit of the medical records of 2,079 HF patients admitted to hospitals in Australia showed that those with coexistent diabetes were less likely to be prescribed a beta-blocker at discharge [odds ratio (OR) 0.721] as compared to those without diabetes [13]. Data from the OPTIMIZEHF registry showed that eligible patients with diabetes were less likely to be prescribed an ACE inhibitor/ARB at discharge ( $81 \%$ vs $83.6 \%$, $\mathrm{p}<0.0001)$ and slightly more likely to receive a beta-blocker $(83.9 \%$ vs $82.6 \%, \mathrm{p}<0.02$ ) [21]. Another study which analyzed HF patients $(\mathrm{N}=3304)$ from nine prospective studies showed that while renin angiotensin aldosterone system (RAAS) blockers and beta-blockers were similarly prescribed in diabetics and non-diabetics $(\mathrm{p}=0.409$ and $\mathrm{p}=0.724$, respectively), diabetics were less likely to receive an aldosterone antagonist as compared with non-diabetics $(p<0.001)$ [25].

It is important to note that despite the high burden of HF patients with comorbid diabetes in India, there is no study that has systematically evaluated differences in the clinical characteristics and management of Indian HF patients with coexistent diabetes vs. those without diabetes.

The HF-DIAB study is a prospective, cross-sectional, multicenter study across India that is aimed at bridging the above-mentioned

research gaps. The research questions for this study are as follows:

Primary Research Question: Is there a difference in the clinical characteristics and the management of chronic HF in Indian outpatients of HF with diabetes vs. those without diabetes?

Secondary Research Question: Is there a difference in the standard treatment prescribed to Indian HF patients with diabetes, as per specialty of the treating doctor (cardiologist vs internist)?

\section{Methods}

\section{General aspects}

The study will recruit a total of 2500 Indian adult outpatient with chronic HF from 250 outpatient clinics across India. It has been initiated in August 2017 and we estimate a period of 10 months for completion of data collection.

\section{Sample size calculation}

The sample size calculation was performed based on the assumption from a previously published study, wherein the percentage of subjects receiving standard therapy for HF (ACE inhibitors/beta-blockers) was $70 \%$ in non-diabetic subjects [18]. The following formula was used to calculate the sample size: $\mathrm{n}=(\mathrm{Za} / 2+\mathrm{Z} \beta) 2 \times(\mathrm{p} 1(1-\mathrm{p} 1)+\mathrm{p} 2(1-\mathrm{p} 2)) /(\mathrm{p} 1-$ $\mathrm{p} 2) 2$, where $\mathrm{p} 1=$ Proportion in one group, $\mathrm{p} 2=$ Proportion in second group, $\alpha=$ Significance level and $(1-\beta)=$ Power.

A total of 860 subjects per group will give a power of $80 \%$ with $5 \%$ level of significance to show a difference of $6 \%$ in non-diabetic subjects receiving ACE inhibitors/beta-blockers as compared with diabetic subjects. Considering a $30 \%$ drop-out and/or withdrawals, a sample size of 1250 subjects per group was considered for the study, resulting in a total sample size of 2500 subjects. This sample size compares favorably with previously published studies.

\section{Participating doctors}

Heart failure is treated by both cardiologists (doctors with a DMCard or DNB-Card degree) and internists [doctors having a degree in internal medicine (MD or DNB-Int Med)]. Since the secondary research question is to ascertain whether any differences in HF management exist between that provided by a cardiologist vs. an internist, approximately 250 cardiologists and 250 internists will be randomly selected from the four main zones (North, South, East and West) of India as study investigators. This will increase representativeness and generalizability of the results. Cipla Ltd. has developed a robust and comprehensive database of doctors in the country over the years, which is periodically verified. A comparison (Table 4 ) of the total number of cardiologists and internists present in the country (as per IMS data: personal communication dated April 22, 2016) versus cardiologists and internists in the Cipla database (data on file, Cipla Ltd.) showed that the Cipla database covers $83 \%$ of the intended doctor pool. Hence, the Cipla database will be used to obtain the list of doctors in the four zones of the country, and the doctors from each zone will be selected in a random manner using SPSS version 11.5 (IBM, New York, U.S.A.) on the basis of proportionate sampling (Figure 1) (Table 5).

\begin{tabular}{|c|c|c|c|}
\hline $\begin{array}{c}\text { Doctor } \\
\text { Specialty }\end{array}$ & $\begin{array}{c}\text { Total number in } \\
\text { country }\end{array}$ & $\begin{array}{c}\text { Total number in } \\
\text { Cipla database }\end{array}$ & $\begin{array}{c}\text { Percentage covered } \\
\text { in Cipla database }\end{array}$ \\
\hline Cardiologists & 7596 & 7000 & $92 \%$ \\
\hline Internists & 23,264 & 18500 & $79.52 \%$ \\
\hline
\end{tabular}

Table 4: Comparison of number of cardiologists and internists in the country with those in the Cipla database. 
Citation: Lopez M, Dalal J, Salvi S, Gogtay J (2018) Study of the Clinical Characteristics and Management of Indian Outpatients of Chronic Heart Failure with Coexistent Diabetes Versus those without Diabetes (HF-DIAB): Rationale and Design. J Cardiovasc Dis Diagn 6: 318. doi: 10.4172/2329-9517.1000318

Page 4 of 6

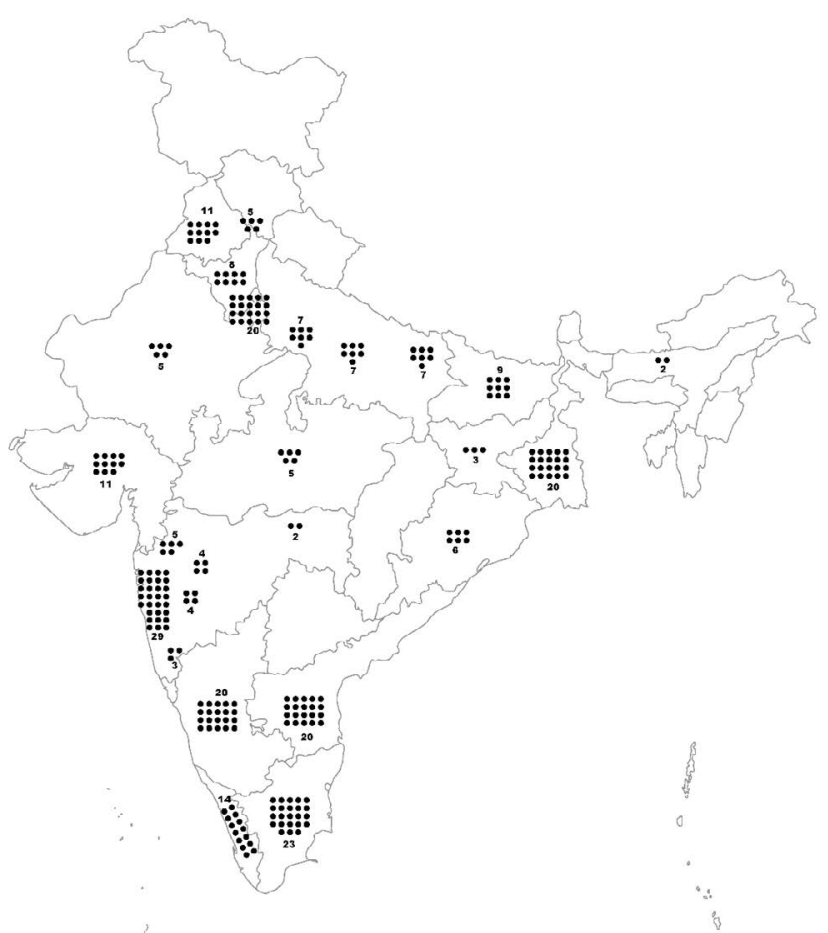

Figure 1: Study sites across India. Number of dots indicate the number of centers.

\begin{tabular}{|c|c|c|c|c|}
\hline \multirow{2}{*}{ Zones } & \multicolumn{2}{|c|}{ Cardiologists } & \multicolumn{2}{c|}{ Internists } \\
\cline { 2 - 5 } & $\begin{array}{c}\text { Percentage } \\
\text { in the Cipla } \\
\text { database }\end{array}$ & $\begin{array}{c}\text { Number } \\
\text { selected for } \\
\text { HF-DIAB study }\end{array}$ & $\begin{array}{c}\text { Percentage } \\
\text { in the Cipla } \\
\text { database }\end{array}$ & $\begin{array}{c}\text { Number } \\
\text { selected for } \\
\text { HF-DIAB study }\end{array}$ \\
\hline East Zone & $15.9 \%$ & 20 & $15.3 \%$ & 19 \\
\hline North Zone & $24.6 \%$ & 31 & $27.4 \%$ & 34 \\
\hline South Zone & $36.5 \%$ & 46 & $25.8 \%$ & 32 \\
\hline West Zone & $23 \%$ & 29 & $31.5 \%$ & 39 \\
\hline Total & $100 \%$ & 126 & $100 \%$ & 124 \\
\hline
\end{tabular}

Table 5: Ratio of doctors zone-wise in the Cipla database and proportionate numbers selected for the HF-DIAB study per zone.

Each participating doctor will be invited to provide reliable information on 5 consecutive outpatients of HF with prior history of diabetes and 5 consecutive outpatients of HF without a prior history of diabetes, thereby generating data on the requisite 2500 patients. A WhatsApp group of the participating doctors will be formed to engage with all the participating doctors. Besides this, personal visits or telephone calls will be made and emails will be sent at regular intervals to the participating doctors to remind them about the study and to encourage study participation and completion.

\section{Patient selection criteria}

Male and female outpatients ( $\geq 18$ years of age) diagnosed with chronic HF based on standard investigations and clinical criteria and confirmed as appropriate for inclusion by the treating physician will be enrolled. Only patients with stable signs and symptoms of HF for at least 3 months will be included in the study. Presence or absence of diabetes will be based on whether the patient has been diagnosed with diabetes prior to study entry. If diabetes is present, the patient will be included only if he/she has had a history of diabetes for at least 6 months.
Hospitalized patients with HF will be excluded from the study. Heart failure patients of the following etiologies will also be excluded: congenital heart disease, valvular heart disease (rheumatic and nonrheumatic) and cor-pulmonale, including primary pulmonary hypertension.

\section{Research tool}

Initial DCF preparation and pilot testing: The draft data capture form (DCF) was prepared based on the available published literature and in keeping with the aims and objectives of the study. It was validated among a group of $25 \mathrm{HF}$ physicians (12 cardiologists and 13 internists) across the four geographical zones of the country and their comments on the DCF were recorded. These comments were then reviewed by two HF specialists, based on which a final DCF (Supplementary File 1) was prepared. This DCF will be the primary research tool for this study.

Key sections of the DCF: It will record the following relevant patient information:

1. Demographics, including age, gender, height, weight, waist circumference and hip circumference.

2. Smoking history in terms of smoking status and number of cigarettes smoked/day.

3. Vital signs: Blood pressure and heart rate.

4. Symptoms/signs of HF: Both current and past signs and symptoms will be recorded.

5. Date of diagnosis of HF.

6. Etiology of HF.

7. Clinical presentation of HF: LVEF, hospitalizations due to HF in the last year and New York Heart Association (NYHA) class will be recorded.

8. Tests done for HF diagnosis.

9. Drugs prescribed for HF: This section will record the drug class, molecule name, dose being taken, duration of treatment, and any previous discontinuation of treatment.

10. Other procedures/interventions done for HF (e.g. PCI, CABG, CRT, ICD)

11. Comorbidities: A history of comorbidities will be elicited but additional diagnostic tests will not be performed to confirm presence of specific comorbidities.

In case of HF patients with coexistent diabetes: the following additional information will be captured:

1. Date of diagnosis of diabetes

2. Recent $\mathrm{HbA} 1 \mathrm{c}$ reading

3. Recent fasting plasma glucose reading

4. Antidiabetic drugs: This section will record the drug class, molecule name, dose being taken, duration of treatment, and any previous discontinuation of treatment.

\section{Study Outcomes}

The primary outcome will be the difference in the percentage of patients receiving a RAAS blocker (ACE inhibitor/ARB/ARNI) and a beta blocker in the HF with coexistent diabetes group versus the HF 
Citation: Lopez M, Dalal J, Salvi S, Gogtay J (2018) Study of the Clinical Characteristics and Management of Indian Outpatients of Chronic Heart Failure with Coexistent Diabetes Versus those without Diabetes (HF-DIAB): Rationale and Design. J Cardiovasc Dis Diagn 6: 318. doi: 10.4172/2329-9517.1000318

Page 5 of 6

without diabetes group. Secondary outcomes will include differences in age, gender, BMI, blood pressure, symptoms, signs and etiology of HF, EF, HF-related hospitalizations, NYHA Class, treatment for $\mathrm{HF}$ and comorbidities in the HF with diabetes group versus the HF without diabetes group. Differences in the treatment of HF between the cardiologist and the internist group will also be evaluated as a secondary outcome.

\section{Data Management and Quality Assurance}

Once the DCFs of the 10 patients are filled, the forms will be sent by each study investigator via a Cipla field representative to the Chest Research Foundation (CRF) for data management and analysis. The database will be designed using the EPI-INFO software version, and fields will be created for all the variables mentioned in the DCF. Dummy entries will be performed for database validation. A double data entry process will be undertaken to help identify transcription errors and discrepancies. Random daily cross-checks of at least $10 \%$ of the questionnaires will be undertaken. Edit check programs will also be written to identify the discrepancies in the entered data. Manual inspection of the data will also be performed to detect errors and inconsistencies. After resolving the discrepancies, data will be coded, locked and the clean data extracted for statistical analysis.

\section{Statistical Analysis}

\section{Demographic characteristics}

The demographic characteristics of the patients will be analysed using descriptive statistics. Continuous variables such as age, blood pressure, etc., will be presented by mean, median, SD, $95 \%$ confidence interval of mean, range (minimum, maximum). Categorical variables such as gender, qualification, etc., will be presented by frequencies and percentages. Graphical presentation will be used for visual representation of the data.

\section{Primary endpoint analysis}

Independent sample t-test will be used to compare the mean difference between diabetic and non-diabetic HF subjects for various clinical characteristics measured using continuous variables. Chisquare test will be used to find out the association between diabetics and non-diabetics with various risk factors and clinical characteristics measured using categorical data. The results will be presented in the form of odds ratio and $95 \%$ confidence interval.

\section{Secondary endpoint analysis}

The Chi-square test will be used to compare the differences in the standard treatment prescribed to Indian HF patients with diabetes according to the specialty of the treating doctor.

Data analysis, tabulation of descriptive statistics will be performed using SPSS 22.0 for Windows. Statistical testing will be performed at the $5 \%$ level of significance using two-tailed tests. Null hypothesis will be rejected if $\mathrm{p}<0.05$.

\section{Deviation from the planned analysis}

Any changes in the planned analysis as described in the protocol (Supplementary File 2) and statistical analysis plan will be discussed in the final study report along with the rationale for the deviation.

\section{Ethics and Dissemination}

The study documents have been reviewed and approved by an independent ethics committee. The study is registered with the Clinical Trials Registry of India (REF/2017/06/014528).

A written, signed and dated data-sharing consent will be obtained by the study investigator from each patient or the patient's legally acceptable representative and impartial witness, as applicable, before enrolment in the study. In order that the participants are given adequate written information about the nature, purpose, possible risks and benefits of the survey in the language that is understood by them, the data-sharing consent form will be made available in English and 11 Indian languages.

The results of the study will be disseminated through publications in peer-reviewed journals.

\section{What this Study Will Add to the Literature}

1. This will be the first ever study on Indian patients of heart failure with coexistent diabetes, comparing this subgroup with HF patients without coexistent diabetes

2. This study will provide a deeper insight into the important subset of Indian HF patients with coexistent diabetes, so that these patients can be more optimally managed and appropriate preventive strategies can be developed

\section{Acknowledgements}

The authors would like to thank Ms. Sapna Madas for assistance in sample size calculation and preparation of the statistical analysis plan. We also thank Ms. Prajakta Gaikwad and Mr. Ninad Sawant for administrative assistance and Mr. Rahul Namjoshi for his inputs.

\section{Contributors}

$\mathrm{ML}$ conceived the study hypothesis. SS, JD, and JG contributed to the study design. ML wrote the protocol; SS, JD and JG provided critical revision of the protocol. ML wrote the manuscript; SS, JD and JG critically reviewed the manuscript. All authors approved the final manuscript.

\section{Funding}

This study was supported by Cipla Ltd. However, the company had no role in the design of the study.

\section{Competing interests}

$\mathrm{ML}$ and JG are employees of Cipla Ltd. The other authors have no competing interests to declare.

\section{References}

1. Ambrosy AP, Fonarow GC, Butler J, Chioncel O, Greene SJ, et al. (2014) The global health and economic burden of hospitalizations for heart failure: lessons learned from hospitalized heart failure registries. J Am Coll Cardiol 63: 11231133.

2. Metra M, Teerlink JR (2017) Heart failure. Lancet 390: 1981-1995.

3. Huffman MD, Prabhakaran D (2010) Heart failure: Epidemiology and prevention in India. Natl Med J India 23: 283-288.

4. Chaturvedi V, Parakh N, Seth S, Bhargava B, Ramakrishnan S, et al. (2016) Heart failure in India: The INDUS (INDia UKieri study) study. J Pract Cardiovasc Sci 2: 28-35.

5. Harikrishnan S (2013). Heart failure in India - Burden and challenges. Health Sciences 2: JS003A.

6. Ponikowski P, Voors AA, Anker SD, Bueno H, Cleland JGF, et al. (2016) 2016 ESC guidelines for the diagnosis and treatment of acute and chronic heart failure. Eur Heart J 37: 2129-2200.

7. Yancy CW, Jessup M, Bozkurt B, Butler J, Casey DE Jr, et al. (2016) 2016 ACC/AHA/HFSA focused update on new pharmacological therapy for heart failure: An update of the 2013 ACCF/AHA guideline for the management of heart failure: A report of the American College of Cardiology/American Hear 
Citation: Lopez M, Dalal J, Salvi S, Gogtay J (2018) Study of the Clinical Characteristics and Management of Indian Outpatients of Chronic Heart Failure with Coexistent Diabetes Versus those without Diabetes (HF-DIAB): Rationale and Design. J Cardiovasc Dis Diagn 6: 318. doi: 10.4172/2329-9517.1000318

Association task force on clinical practice guidelines and the Heart Failure Society of America. J Am Coll Cardiol 68: 1476-1488.

8. Komajda M, Cowie MR, Tavazzi L, Ponikowski P, Anker SD, et al. (2017) Physicians' guideline adherence is associated with better prognosis in outpatients with heart failure with reduced ejection fraction: the QUALIFY international registry. Eur J Heart Fail 19: 1414-1423.

9. Yoo BS, Oh J, Hong BK, Shin DH, Bae JH, et al. (2014) Survey of Guideline Adherence for Treatment of Systolic Heart Failure in Real World (SUGAR): a multi-center, retrospective, observational study. PLoS One 9: e86596.

10. Harikrishnan S, Sanjay G, Agarwal A, Kumar NP, Kumar KK, et al. (2017) Oneyear mortality outcomes and hospital readmissions of patients admitted with acute heart failure: Data from the Trivandrum Heart Failure Registry in Kerala, India. Am Heart J 189: 193-199.

11. Pokharel Y, Wei J, Hira RS, Kalra A, Shore S, et al. (2016) Guideline-directed medication use in patients with heart failure with reduced ejection fraction in India: American College of Cardiology's PINNACLE India Quality Improvement Program. Clin Cardiol 39: 145-149.

12. Jankowska EA, Kalicinska E, Drozd M, Kurian B, Banasiak W, et al. (2014) Comparison of clinical profile and management of outpatients with heart failure with reduced left ventricular ejection fraction treated by general practitioners and cardiologists in contemporary Poland: the results from the DATA-HELP registry. Int J Cardiol 176: 852-858.

13. Wlodarczyk JH, Keogh A, Smith K, McCosker C (2003) CHART: congestive cardiac failure in hospitals, an Australian review of treatment. Heart Lung Circ 12: $94-102$.

14. Komajda M, Follath F, Swedberg K, Cleland J, Aguilar JC, et al. (2003) The EuroHeart Failure Survey programme--a survey on the quality of care among patients with heart failure in Europe. Part 2: treatment. Eur Heart J 24: 464-474.

15. Dei Cas A, Fonarow GC, Gheorghiade M, Butler J (2015) Concomitant diabetes mellitus and heart failure. Curr Probl Cardiol 40: 7-43.

16. Cohen-Solal A, Beauvais F, Logeart D (2008) Heart failure and diabetes mellitus: Epidemiology and management of an alarming association. J Cardiac Fail 14: 615-625
17. Dokainish H, Teo K, Zhu J, Roy A, Al-Habib KF, et al. (2017) Global mortality variations in patients with heart failure: results from the International Congestive Heart Failure (INTER-CHF) prospective cohort study. Lancet Glob Health 5 : e665-672.

18. Lam CS, Teng TK, Tay WT, Anand I, Zhang S, et al. (2016) Regional and ethnic differences among patients with heart failure in Asia: the Asian sudden cardiac death in heart failure registry. Eur Heart J 37: 3141-3153.

19. Aguilar D, Deswal A, Ramasubbu K, Mann DL, Bozkurt B (2010) Comparison of patients with heart failure and preserved left ventricular ejection fraction among those with versus without diabetes mellitus. Am J Cardiol 105: 373-377.

20. Melgaard L, Gorst-Rasmussen A, Søgaard P, Rasmussen LH, Lip GY, et al. (2016) Diabetes mellitus and risk of ischemic stroke in patients with heart failure and no atrial fibrillation. Int J Cardiol 209: 1-6.

21. Greenberg BH, Abraham WT, Albert NM, Chiswell K, Clare R, et al. (2007) Influence of diabetes on characteristics and outcomes in patients hospitalized with heart failure: a report from the Organized Program to Initiate Lifesaving Treatment in Hospitalized Patients with Heart Failure (OPTIMIZE-HF). Am Heart J 154: 277.e1-e8.

22. Shi C, Wang LJ, Hu DF, Li JP, Zhu TQ, et al. (2010) Prevalence, clinica characteristics and outcome in patients with chronic heart failure and diabetes. Chin Med J 123: 646-650.

23. Cubbon RM, Adams B, Rajwani A, Mercer BN, Patel PA, et al. (2013) Diabetes mellitus is associated with adverse prognosis in chronic heart failure of ischaemic and non-ischaemic etiology. Diab Vasc Dis Res 10: 330-336.

24. Dauriz M, Targher G, Laroche C, Temporelli PL, Ferrari R, et al. (2017) ESC-HFA Heart Failure Long-Term Registry. Association between diabetes and 1-year adverse clinical outcomes in a multinational cohort of ambulatory patients with chronic heart failure: Results from the ESC-HFA Heart Failure Long-Term Registry. Diabetes Care 40: 671-678.

25. Edelmann F, Wachter R, Düngen HD, Stork S, Richter A, et al. (2011) Heart failure therapy in diabetic patients-comparison with the recent ESC/EASD guideline. Cardiovasc Diabetol 10: e1-e8. 